\title{
Comparison of catheter-associated bacteriuria and urinary tract infections between designed sheathed urethral catheters versus other methods of urinary drainage
}

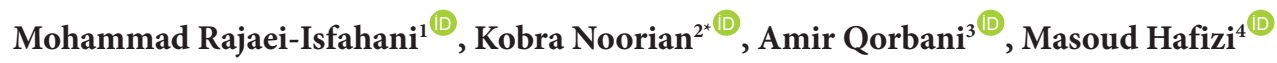 \\ ${ }^{1}$ Department of Urology, Shahrekord University of Medical Sciences, Shahrekord, Iran \\ ${ }^{2}$ Shahrekord University of Medical Sciences, Shahrekord, Iran \\ ${ }^{3}$ Department of Pathology and Laboratory Sciences, University of California, San Francisco (UCSF), USA \\ ${ }^{4}$ Department of Infectious Diseases, Shahrekord University of Medical Sciences, Shahrekord, Iran
}

\section{A R T I C L E I N F O}

Article Type:

Original

\section{Article History:}

Received: 17 March 2020

Accepted: 28 June 2020

Published online: 21 July 2020

Keywords:

Catheter-associated urinary tract infection

Designed sheathed catheter

Bacteriuria

\begin{abstract}
A B S T R A C T
Introduction: One of the most common nosocomial infections is urinary tract infection (UTI) which more commonly occurs in patients with urethral catheter.

Objectives: The aim of this study is to compare the incidence rate of catheter-associated bacteriuria (CRB) and UTI between sheathed designed catheters versus other methods of urinary drainage.

Patients and Methods: In this randomized double-blind controlled trial, 150 male patients, who needed urethral catheter, were randomly assigned into three groups; condom bag, designed sheath catheter and traditional Foley catheter. The midstream urine samples were sent to laboratory to evaluate bacteriuria immediately after catheter insertion, upon catheter removal and after two weeks consequently.

Results: The mean age of patients was $51.28 \pm 14.12$ years. The frequency of bacteriuria in patients was $19(38 \%), 10(20 \%)$ and $10(20 \%)$ in the traditional catheter, condom bag and sheathed designed catheter, respectively, which was strongly significant $(P<0.001)$.

Conclusion: The frequency of bacteriuria in the patients with designed sheathed catheter was significantly less than the traditional catheter. Therefore, using the designed catheter in short-term may be safer in the patients.

Trial Registration: The trial was registered in the Thai Clinical Trials Registry (identifier: TCTR20200703002; http://www.clinicaltrials.in.th/index.php?tp=regtrials\&menu=trialsear ch\&smenu=fulltext\&task=search\&task2=view1\&id=6443).
\end{abstract}

Implication for health policy/practice/research/medical education:

In a randomized double-blind controlled trial on 150 male patients, who needed urethral catheter, we found that designed sheathed catheter can reduce the incidence of bacteriuria in a short-term period compared to the traditional Foley urethral catheter. Therefore, we suggest using the designed sheathed catheter in hospitalized patients, especially those in ICU.

Please cite this paper as: Rajaei-Isfahani M, Noorian K, Qorbani A, Hafizi M. Comparison of catheter-associated bacteriuria and urinary tract infections between designed sheathed urethral catheters versus other methods of urinary drainage. J Renal Inj Prev. 2022; 11(1): e03. doi: 10.34172/jrip.2022.03.

\section{Introduction}

A hospital-acquired infection, also known as nosocomial infection, is one of the most common causes of morbidity and mortality in hospitalized patients (1). The prevalence of nosocomial infection in hospitalized patients is mentioned to be $4 \%-47 \%$ that is accounted for about $80 \%$ of deaths associated with nosocomial infections (2). Urinary tract infection (UTI) constitutes nearly $40 \%$ of nosocomial infections and about $80 \%$ of such infections are due to the urinary tract catheterization $(3,4)$. Additionally $15 \%$ $25 \%$ of patients admitted in the emergency department undergo urinary tract catheter since more than half of them develop bacteriuria within five days (5). The incidence of bacteriuria in hospitalized adults and elderly with urethral catheter increases $1-3 \%$ and $15 \%$ per day, respectively. Twenty percent of patients with bacteriuria 
show symptomatic UTI (4). Some of the predisposing factors of UTI are female gender, old age, prolonged antibiotic administration and immunosuppression (6). Several strategies are recommended to reduce the risk of UTI in patients with urinary tract catheter, including; healthcare personnel education, hand washing and sterilization before the procedure, frequently changing the catheter and avoiding unnecessary catheterization (7).

Several studies were conducted on different methods of reducing the incidence rate of UTI in the patients with urinary catheter. Koskeroglu et al showed that the use of meatal disinfectant is not effective in preventing the catheter-related bacteriuria (CRB) (8). The study by Waites et al showed that bladder washout with acetic acid, neomycin-polymyxin or normal saline does not have significant antibacterial effects on the CRB (9). However, some studies showed that daily usage of povidone iodine (betadine) might be effective in preventing the CRB (10). Microorganisms mainly enter the bladder by migrating along with the space between external surface of the catheter and urethra (extraluminal route) or less commonly directly through the catheter (intraluminal route) (11).

\section{Objectives}

As mentioned above, prior studies were controversial and no successful method was proven in preventing CRB. Therefore, the aims of this study were to evaluate the effects of designed sheathed catheter in preventing the entrance of microorganisms from outer surface of catheter into the bladder by applying mechanical and chemical barriers to urinary catheter and to compare its effects with routine catheterization and condom bag.

\section{Patients and Methods}

In this randomized double-blind controlled trial, 150 patients (circumcised male) who were admitted to Hajar and Kashani hospital were enrolled. Inclusion criteria were: all the hospitalized patients in urology, intensive care unit, cardiac care unit, surgery and neurology units who needed urinary catheterization and who were older than 18 years. Exclusion criteria were having a positive urinary culture at the time of catheterization, current antibiotic consumption or having any kind of urology operation during the study. The patients were randomly assigned into three equal groups; convectional catheters (control group), condom-bag and designed catheter (case group) (Figure 1). In the first group (control), the common latex Foley 16-18fr catheter was inserted after sterilizing the external genitalia. In the second group condom bag was used in patients with urinary incontinence after sterilizing the external genitalia. In the third group after sterilizing the external genitalia, designed sheathed catheter was used, which was a sterilized Foley catheter with a designed condom on external genitalia as a sheath. In all the patients, the midstream urine samples were sent to the laboratory for urinalysis and urine culture on three separate times, after catheter insertion, at the time of catheter removal and after two weeks of removal. Positive urine culture was defined as colony count of $\geq 1000 / \mathrm{cfu} /$ $\mathrm{mL}$ (1). Ten milliliters of urine sample were taken from Foley catheter or condom using syringe after serializing

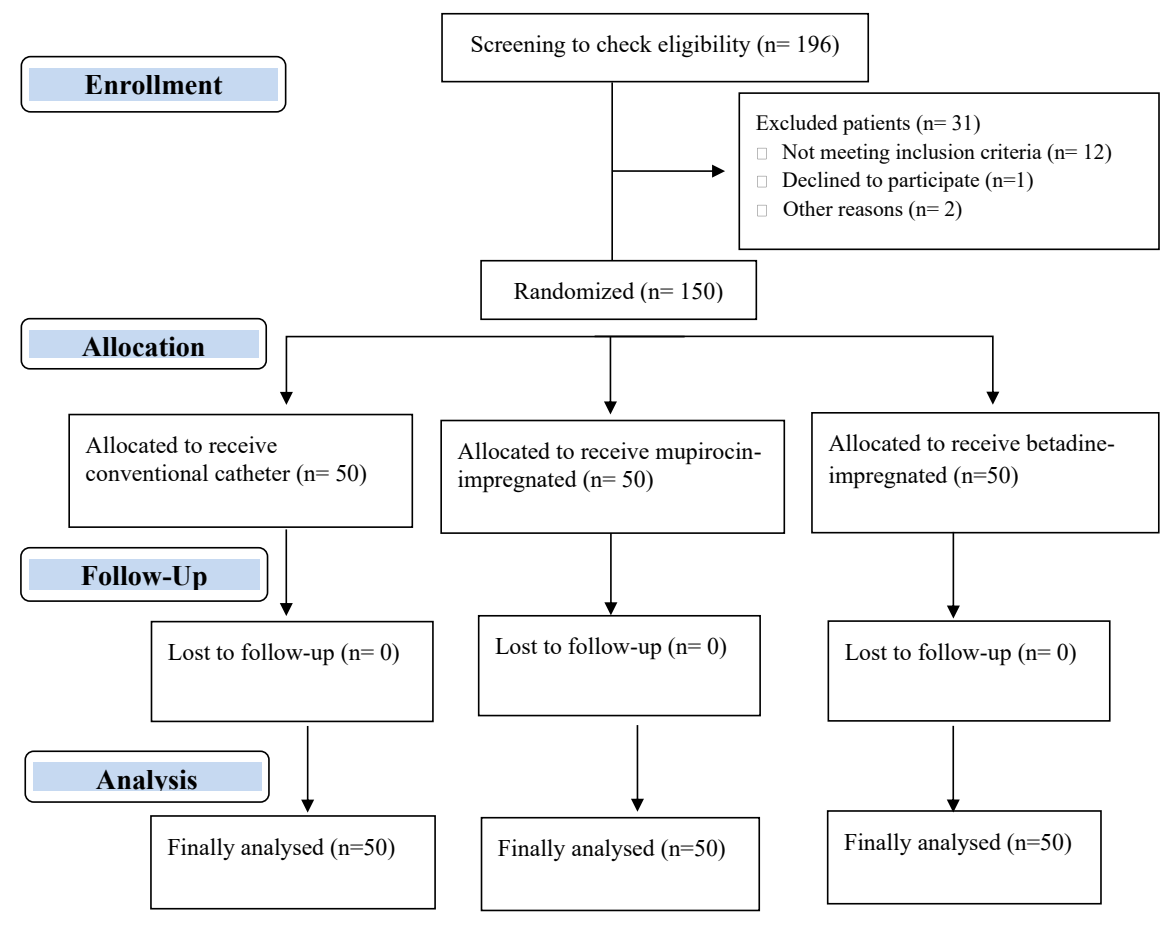

Figure 1. CONSORT flow chart showing the flow of patients through the trial. 
the tap location. All urinary tests were analyzed in the same laboratory using the same methods. Demographic information including age, gender, ward and method of catheterization, urine analysis, urethra catheter and also duration of catheterization were recorded and analyzed. The validity of this tool was obtained using content validity.

\section{Statistical analysis}

Descriptive statistics (frequency, mean and standard deviation) and analytical statistics were used to analyse the data anlysis. All the analysis was conducted using SPSS. The Kolmogorov-Smirnov statistical test demonstrated that the data did not have normal distribution. Thus, the nonparametric tests were used in order to analyze the data. $P<0.05$ was considered statistically significant.

\section{Results}

Around, 150 patients were participated in this study, with the mean age of $51.28 \pm 14.12$ years. No significant age difference observed between the three groups. The duration of catheterization was between 3-10 days without any significant difference between the three groups $(P>0.05)$. The number of patients in neurology, urology, ICU, CCU, surgery wards were 21, 25, 37, 26 and 41 patients, respectively.

The prevalence of UTI was compared between the three groups at the discharge time and after two weeks of discharge. Incidence of UTI was significantly greater in the control group compared to the other two groups (19 versus 10 and 10 cases; $P<0.001$ ) but there was no significant difference between the two groups regarding the incidence of UTI after two weeks $(P>0.05)$. The results also showed that there was no significant difference between condom bag and designed sheathed catheter groups at discharge time and two weeks after the study (Table 1).

At the time of discharge, 39 patients (26\%) had UTI. E. coli was the most common cause of UTI, accounting for infection of 23 out of 36 patients. Three patients experienced UTI due to pseudomonas and others (10 patients) had both E. coli and pseudomonas positive cultures. UTI in three patients was related to other microbial agents (Table 2).

There was a significant relationship between the incidence of UTI and the patients' age $(P<0.001)$. Additionally, the results of independent t-test showed a significant difference between the mean duration of catheterization and the incidence rate of UTI $(P<0.001$; Table 3).

The main catheterization associated side effects are listed in Table 4 (fever, flank pain, catheter obstruction, dysuria, hematuria, wound and urinary obstruction). Although the most common complaint was dysuria (28\%) which was reported in designed sheathed catheter, however, the overall reported complications were reported in the control group ( $40 \%$ of patients) compared to condom catheter (28\%) and designed sheathed catheter (30\%).

\section{Discussion}

The most common nosocomial infection is catheterassociated infection. This study showed that using designed sheathed catheter with mechanical barrier could reduce bacteriuria in patients with permanent urinary catheter compared to the conventional urinary catheter. Prior studies showed controversial results in applying different methods on reducing catheter-associated infection. Parker et al evaluated the effect of nitrofurazone coated catheter and hydrogel latex in reducing the catheter-related infection, which showed no significant difference between the two methods (12). In a study on patients with urethral catheter, Schumm et al showed that silver alloy urethral catheter could reduce bacteriuria in a short-term period compared to silver oxide catheter, however the results did not show a significant difference in one-week post catheterization follow up (13).

Drekonja et al evaluated the effects of antibacterial catheter in reducing catheter-related infection. They

Table 1. Incidence rate of urinary tract infection (UTI) in studied groups

\begin{tabular}{|c|c|c|c|c|}
\hline \multirow[b]{2}{*}{ Times } & \multicolumn{3}{|c|}{ Groups } & \multirow[b]{2}{*}{$P$ value } \\
\hline & $\begin{array}{c}\text { Group } 1 \\
\text { (convectional catheter) }\end{array}$ & $\begin{array}{c}\text { Group } 2 \\
\text { (Condom bag catheter) }\end{array}$ & $\begin{array}{c}\text { Group } 3 \\
\text { (Designed sheathed catheter) }\end{array}$ & \\
\hline Discharge time & $19(38 \%)$ & $10(20 \%)$ & $10(20 \%)$ & $<0.001$ \\
\hline After 2-week & $2(4 \%)$ & $2(4 \%)$ & $3(6 \%)$ & $>0.05$ \\
\hline
\end{tabular}

Table 2. Microbial cause of urinary tract infection in studied groups

\begin{tabular}{|c|c|c|c|c|}
\hline \multirow{2}{*}{ Catheterization } & \multicolumn{4}{|c|}{ Microbial agents } \\
\hline & Mix (E. coil \& Pseudomonas) & E. coil & Pseudomonas & Other \\
\hline Conventional method & $3(6 \%)$ & $14(28 \%)$ & $1(2 \%)$ & $1(2 \%)$ \\
\hline Condom bag catheter & $2(4 \%)$ & $6(12 \%)$ & $1(2 \%)$ & $1(2 \%)$ \\
\hline Designed sheathed catheter & $5(10 \%)$ & $3(6 \%)$ & $1(2 \%)$ & $1(2 \%)$ \\
\hline
\end{tabular}


Rajaei-Isfahani $M$ et al

Table 3. Effect of patient's age and duration of catheterization on urinary tract infection rate

\begin{tabular}{llccc}
\hline & & Frequency & Mean & Standard deviation \\
\hline \multirow{2}{*}{ Age (year) } & Uninfected & 111 & 48.4 & 14.923 \\
& Infected & 39 & 55.4 & 14.520 \\
\multirow{2}{*}{ Catheterization duration (day) } & Uninfected & 111 & 3.71 & 1.494 \\
& Infected & 39 & 5.46 & 2.286 \\
\hline
\end{tabular}

Table 4. The incidence rate of catheterization-related side effects in different groups

\begin{tabular}{|c|c|c|c|c|c|c|c|c|}
\hline \multirow{2}{*}{ Method } & \multirow[b]{2}{*}{ Fever } & \multicolumn{7}{|c|}{ Complication } \\
\hline & & Flank pain & Catheter obstruction & Dysuria & Hematuria & Wound & Obstruction tract & Fever \\
\hline \multirow{2}{*}{ Conventional method } & No. & 5 & 6 & 3 & 18 & 6 & 1 & 1 \\
\hline & $\%$ & 3.3 & 4 & 2 & 12 & 4 & 0.66 & 0.66 \\
\hline \multirow{2}{*}{ Condom bag catheter } & No. & 2 & 2 & 0 & 10 & 3 & 4 & 0 \\
\hline & $\%$ & 1.3 & 1.3 & 0 & 6.6 & 2 & 2.6 & 0 \\
\hline \multirow{2}{*}{ Designed sheathed catheter } & No. & 6 & 6 & 2 & 14 & 3 & 4 & 0 \\
\hline & $\%$ & 4 & 4 & 1.3 & 9.3 & 2 & 2.6 & 0 \\
\hline \multirow{2}{*}{ Total } & No. & 13 & 14 & 5 & 42 & 12 & 9 & 1 \\
\hline & $\%$ & 8.6 & 9.3 & 3.33 & 28 & 8 & 6 & 0.66 \\
\hline
\end{tabular}

reported that antibacterial catheters were effective in reducing bacteriuria and funguria, however it is not effective in reducing the incidence rate of symptomatic UTI (14). In contrast to the study by Drekonja et al, Koskeroglu et al showed that use of disinfectant is ineffective in reducing bacteriuria (8). These differences in results could be due to the different study population such as presence or absence of circumcision that may influence the incidence of UTI.

In our study, the incidence rate of catheter-related bacteriuria was $26 \%$ that was nearly similar to the result of the study by Haley et al (15). In this regard, Edmond et al showed that $40 \%$ of nosocomial infections were related to UTI while $80 \%$ of cases were related to urinary catheterization (16). The duration of urinary catheterization is an important factor in catheter-related bacteriuria $(17,18)$. Parker et al reported that the incidence of bacteriuria in hospitalized adults and elderly patients with urethral catheter increases to $1-3 \%$ and $15 \%$ per day respectively. Thereby, the incidence rate of bacteriuria after 7 and 14 days of catheterization will increase to 35\% and $75 \%$ respectively (4). These findings show that we can decrease the catheter-related infection by reducing the duration of urinary catheterization (19-21). Our findings showed that the incidence rate of condom catheter infection was 20\% and traditional Foley catheter was 38\%. Several studies, such as Saint et al and Ouslander et al studies also reported that the use of condom catheters is less likely to lead to bacteriuria, symptomatic UTI or death than the use of indwelling catheters $(22,23)$. However, genital injury is more commonly seen in condom catheters (24). The most common cause of bacteriuria in our study was Escherichia-coli, which was in line with other studies such as Wu et al (17).

\section{Conclusion}

In this study, we found that designed sheathed catheter can reduce the incidence of bacteriuria in a short-term period compared to the traditional Foley urethral catheter. Therefore, we suggest using the designed sheathed catheter in hospitalized patients, especially those in ICU.

\section{Limitations of the study}

In this study, all of the patients were Muslim male who were circumcised. Therefore, it could have an impact on the catheter related-infection in the patients who used condom or designed sheathed catheter. Other limitations of the study were the small sample size and the short time of follow-up. Therefore, we recommend conducting further studies using larger sample size and longer followup.

\section{Authors' contribution}

MRI conducted the research. MH gathered the data. KN analyzed the data. MRI and MH prepared the primary draft. AQ contributed to writing the manuscript and review of articles. KN edited the manuscript. KN prepared the final paper. All authors read and seen the final manuscript.

\section{Conflicts of interest}

The authors declare no conflict of interest.

\section{Ethical issues}

The research followed the tenets of the Declaration of Helsinki. This paper was extracted from the MD student thesis of Amir Qorbani, in Kashani Hospital, School of Medicine, Shahrekord University of Medical Sciences. The study protocol was registered in the Registry of 
Clinical Trials (identifier: TCTR20200703002; http:// www.clinicaltrials.in.th/index.php?tp=regtrials\&men $\mathrm{u}=$ trialsearch \&smenu $=$ fulltext \&task $=$ search\&task $2=\mathrm{v}$ iew1\&id=6443). Moreover, the study was approved by the ethics committee of the Shahrekord University of Medical Sciences (\#739). Accordingly, informed consent was obtained from all the patients. Moreover, ethical issues (including plagiarism, data fabrication, double publication) have been completely observed by the authors.

\section{Funding/Support}

We hereby gratefully thank the Research and Technology Deputy of the Shahrekord University of Medical Sciences and all the people who helped us to conduct this study. Shahrekord University of Medical Sciences supported funding for this study.

\section{References}

1. Hooton TM, Bradley SF, Cardenas DD, Colgan R, Geerlings SE, Rice JC, et al. Diagnosis, prevention, and treatment of catheter-associated urinary tract infection in adults: 2009 International Clinical Practice Guidelines from the Infectious Diseases Society of America. Clin Infect Dis. 2010;50:625-63. doi: $10.1086 / 655159$

2. Igawa Y, Wyndaele JJ, Nishizawa O. Catheterization: possible complications and their prevention and treatment. Int J Urol. 2008;15:481. doi: 10.1111/j.14422042.2008.02075.x

3. Lam TBL, Omar MI, Fisher E, Gillies K, MacLennan S. Types of indwelling urethral catheters for short-term catheterization in hospitalized adults. Cochrane Database Syst Rev. 2014;(9):CD004013. doi: 10.1002/14651858. CD004013.pub4.

4. Parker D, Callan L, Harwood J, Thompson DL, Wilde, M, Gray M. Nursing interventions to reduce the risk of catheter-associated urinary tract infection. J Wound Ostomy Continence Nurs. 2009;36:23-34. doi: 10.1097/01. WON.0000345173.05376.3e.

5. Parida S, Mishra SK. Urinary tract infections in the critical care unit: A brief review. Indian J Crit Care Med. 2013;17(6):370-374. doi: 10.4103/0972-5229.123451.

6. Smith JM. Indwelling catheter management: From habit-based to evidence-based practice. Ostomy Wound Manage.2003; 49:34-45.

7. Parry MF, Grant B, Sestovic M. Successful reduction in catheter-associated urinary tract infections: Focus on nurse-directed catheter removal. Am J Infect Control. 2013;41:1178-81. doi: 10.1016/j.ajic.2013.03.296.

8. Koskeroglu N, Durmaz G, Bahar M, Kural M, Yelken B. The role of meatal disinfection in preventing catheterrelated bacteriuria in an intensive care unit: a pilot study in Turkey. J Hosp Infect. 2004;56:236-8. doi: 10.1016/j. jhin.2003.12.017

9. Waites KB, Canupp KC, Roper JF, Camp SM, Chen Y. Evaluation of 3 method of bladder irrigation to treat bacteriuria in persons with neurogenic bladder. J Spinal Cord. 2006;29:217-26.

10. Chundamala J, Wright JG. The efficacy and risks of using povidone-iodine irrigation to prevent surgical site infection: an evidence-based review. Can J Surg. 2007;50:473-481.

11. Maki DG, Tambyah PA. Engineering out the risk for infection with urinary catheters. Emerg Infect Dis. 2001;7:342-347. doi:10.3201/eid0702.010240

12. Parker D, Callan L, Harwood J, Thompson DL, Wilde M, Gray M. Nursing interventions to reduce the risk of catheter-associated urinary tract infection. Part 1: Catheter selection. J Wound Ostomy Continence Nurs. 2009;36:2334. doi: 10.1097/01.WON.0000345173.05376.3e.

13. Schumm K, Lam TB. Types of urethral catheters for management of short-term voiding problems in hospitalized adults: a short version Cochrane review. Neurourol Urodyn. 2008;27:738-46. doi: 10.1002/14651858.CD004013.pub3

14. Drekonja DM, Kuskowski MA, Wilt TJ, Johnson JR. Antimicrobial urinary catheters: a systematic review. Expert Rev Med Devices. 2008;5:495-506. doi: 10.1586/17434440.5.4.495.

15. Haley RW, Hooton TM, Culver DH, Stanley RC, Emori TG, Hardison CD, et al. Nosocomial infections in U.S. hospitals, 1975-1976: estimated frequency by selected characteristics of patients. Am J Med. 1981;70:947-59.

16. Edmond MB, Wallace SE, McClish DK, Pfaller MA, Jones RN, Wenzel RP. Nosocomial bloodstream infections in United States hospitals: a three-year analysis. Clin Infect Dis. 1999;29:239-44. doi: 10.1086/520192

17. Wu CJ, Lee HC, Lee NY. Predominance of Gram-negative bacilli and increasing antimicrobial resistance in nosocomial bloodstream infections at a university hospital in southern Taiwan. J Microbiol Immunol Infect. 2006; 39:135-43.

18. Janzen J, Buurman BM, Spanjaard L, de Reijke TM, Goossens A, Geerlings SE. Reduction of unnecessary use of indwelling urinary catheters. BMJ Qual Saf. 2013;22:984-8. doi: 10.1136/ bmjqs-2013-001908.

19. Maki DG. A prospective study of the pathogenesis of catheter-associated urinary tract infection. Mayo Clin Proc. 1999;74:131-6. doi:10.4065/74.2.131

20. Bruun JN, Digranes A. Bladder irrigation in patients with indwelling catheters. Scand J Infect Dis. 1978;10:71-74. doi:10.3109/inf.1978.10.issue-1.16

21. Al-Hazmi H. Role of duration of catheterization and length of hospital stay on the rate of catheter-related hospitalacquired urinary tract infections. Res Rep Urol. 2015;7:41-7. doi: $10.2147 /$ RRU.S75419

22. Saint S, Kaufman SR, Rogers MA, Baker PD, Ossenkop K, Lipsky BA. Condom versus indwelling urinary catheters: a randomized trial. J Am Geriatr Soc. 2006;54:1055-61. doi:10.1111/j.1532-5415.2006.00785.x

23. Ouslander JG, Greengold BA, Silverblatt FJ. An accurate method to obtain urine for culture in men with external catheters. Arch Intern Med 1987;147:286-8.

24. Johnson JR, Kuskowski MA, Wilt TJ. Systematic review: antimicrobial urinary catheters to prevent catheter-related urinary tract infection in hospitalized patients. Ann Intern Med. 2006;144:116-26.

Copyright $\odot 2022$ The Author(s); Published by Nickan Research Institute. This is an open-access article distributed under the terms of the Creative Commons Attribution License (http://creativecommons.org/licenses/by/4.0), which permits unrestricted use, distribution, and reproduction in any medium, provided the original work is properly cited. 